\title{
Venous thromboembolism is a relevant and underestimated adverse event in cancer patients treated in phase I studies
}

\author{
M Mandalä, I, F Grosso ${ }^{2}$, C Vitalini ${ }^{3}$, I Corradino ${ }^{3}$, R Sanfilippo ${ }^{4}$, S Colombini ${ }^{3}$, M Clerici ${ }^{3}$, R Labianca', \\ A De Pascale ${ }^{3}$ and $S$ Marsoni $^{3}$
}

'Division of Medical Oncology, Department of Oncology and Hematology, Ospedali Riuniti, Largo Barozzi I, Bergamo 24I 28, Italy; ${ }^{2}$ Department of Oncology and Haematology, Ospedale Santi Antonio e Biagio, 15/21 Alessandria, Italy; ${ }^{3}$ SENDO Foundation, 20122 Milan, Italy; ${ }^{4}$ Adult Sarcoma Medical Oncology Unit, Cancer Medicine Department, National Cancer Institute, 20133 Milan, Italy

BACKGROUND: To investigate, retrospectively, the role of tumour histotype and antiangiogenic drugs for venous thromboembolism (VTE) development in advanced cancer patients treated in phase I studies.

METHODS: Patients enrolled and treated in phase I studies conducted by SENDO (Southern Europe New Drugs Organisation) were considered.

RESULTS: Data of 1415 patients were included in the analysis: 526 (37.2\%) patients were males, median age was 57.3 years (range: 13-85). Fifty-six (3.96\%) patients developed a VTE. At multivariate analysis gynaecologic (hazard ratio (HR): 2.8, 95\% confidence interval $(\mathrm{Cl})$ : $1.29-6.23, P=0.009$ ) and gastrointestinal tumours (HR: $3.23,95 \% \mathrm{Cl}:$ I.I8-8.87, $P=0.023$ ) as well as combination regimens of cytotoxic and antiangiogenic agents (HR: 2.6, 95\% Cl: $|$.$| I I-6.30, P=0.028$ ), white blood cell $>\left.|| 000 \mu\right|^{-1}$ (HR: 2.59, 95\% Cl: $1.10-6.09, P=0.028)$ and haemoglobin $<10 \mathrm{~g} \mathrm{dl}^{-1}(\mathrm{HR}: 3.1,95 \% \mathrm{Cl}: 1.07-8.94, P=0.037)$ were statistically correlated with VTE development. Venous thromboembolism was the fourth most common cause of drug discontinuation. The median time from first drug administration to discontinuation was 1.4 for VTE and 2.3 months for the other adverse events $(P=0.02)$.

CONCLUSION: Venous thromboembolism is a relatively common complication among patients treated in the context of phase I studies, and may lead to early drug discontinuation. A greater risk of developing VTE is associated with the diagnosis of gynaecologic and gastrointestinal tumours and the combined use of chemotherapy and antiangiogenic drugs. British Journal of Cancer (2012) 107, 612-616. doi:10.1038/bjc.2012.325 www.bjcancer.com Published online 24 July 2012

(c) 2012 Cancer Research UK

Keywords: venous thromboembolism; phase I studies; adverse event

Phase I studies are designed primarily to identify the maximum tolerated dose (MTD) for further clinical development of new drugs, and to evaluate their tolerability and toxicity profile. They include first-in-human studies as well as studies that combine two or more experimental or previously approved drugs for the first time (Maur and Sessa, 2007).

The clinical benefits of Phase I trials are limited, whereas toxicity-related risks in patients entering these studies may be considerable. Additionally, patients who are considered for these studies are usually in a vulnerable position, having advanced malignancies with no standard therapeutic options.

The assessment of dose-limiting toxicities (DLTs) is critical in phase I cancer clinical trials, as this represents the primary end point to determine the MTD, and an accurate reporting of safety data is crucial in the early phase of drug development.

In cancer patients, venous thromboembolism (VTE) is a relevant cause of morbidity and mortality negatively impacting on quality of life and health-care costs (Kahn et al, 2005). Cancer is a well-recognised independent risk factor for VTE, with almost one-fifth of all new VTE events associated with active cancer.

*Correspondence: Dr M Mandalà; E-mail: mariomandala@tin.it Received 19 March 2012; revised 30 May 2012; accepted 31 May 2012; published online 24 July 2012
Cancer patients have a 4- to 7-fold higher risk for VTE than patients without cancer, and about $10 \%$ of them will suffer a VTE episode during their life time, mostly while on chemotherapy (Falanga and Zacharski, 2005).

Despite the general perception that the incidence of VTE complications in metastatic patients is a high, few data are available for patients participating in phase I studies, in particular concerning the possible influence of tumour histotype and type of treatment (Vemulapalli et al, 2009; Mandala et al, 2012). Furthermore, there is no information regarding the relevance and the timing of VTE in the context of the commonly reported toxicities.

In a recent review evaluating how DLTs have been defined in oncology phase I trials of molecular target agents, VTE was never found to be part of any DLT definition (Le Tourneau et al, 2011).

The ideal setting to investigate this issue is a prospective database in which clinical data have been prospectively collected during the studies, including VTE.

In a previous paper, we validated the Khorana's score in patients treated in phase I studies (Mandala et al, 2010). In the present paper, we investigate the role of tumour histotype and chemotherapy with antiangiogenic drugs for the VTE development. Furthermore, we evaluate the relevance of VTE in the context of the commonly reported haematologic and non-haematologic toxicities. 


\section{PATIENTS AND METHODS}

The trials and patients database, consisting of 1415 patients enrolled between 2000 and 2010 in 15 centres participating in the Southern Europe New Drugs Organisation (SENDO) Foundation phase I trials, have been previously described in detail (Mandala et al, 2012). In the current study, we considered all adverse events (AEs) occurring from the first dose of the study treatment up to 30 days after treatment discontinuation. All AEs were reviewed by the SENDO Medical Officer.

The study population was classified according to demographic and cancer-related characteristics (age, gender, tumour type) and to the type of antitumour therapy received in the study (cytotoxic drugs and/or different types of target therapies).

To investigate a possible difference in the time to progression and objective response rate between patients with and without VTE, a random selection of patients not experiencing VTE was performed and represented the control group: for each patient with VTE, two patients without VTE were matched according to tumour type, age $( \pm 5)$, gender and type of antineoplastic treatment (e.g., cytotoxic therapy, antiangiogenic/antivascular/other target therapy, combination of two different treatment modalities).

All the AEs, including VTE, were prospectively collected during the study protocols.

In all studies but one requiring prophylaxis with acetylsalicylic acid no primary antithrombotic prophylaxis was foreseen during treatment but anticoagulants at thromboprophylaxis dose were allowed at investigator's discretion.

The criterion to diagnose VTE by compression US was noncompressibility of a proximal vein. When symptoms suggestive for pulmonary embolism developed, a radionuclide lung scanning, a CT scan or both were performed.

\section{Statistical methods}

Descriptive statistics were applied to the study population to present demographic and clinical characteristics: median, mean, range for continuous variables; number and percentage for qualitative variables.

No stratification by study was applied because of the very limited sample sizes of most of these studies and of the low incidence of VTEs.

Univariate analyses were performed on variables identified as potential risk factors, that is, tumour and treatment type, white blood cells (WBC), platelet count and haemoglobin, by applying the log-rank test. All of these variables were incorporated in the multivariate proportional Cox model with sex and age, including platelet count, which was the only variable associated with a $P$-value of $>0.05$, but was nevertheless recognised as a known risk factor.

The results were reported as hazard ratios (HRs) with $95 \%$ confidence intervals ( $95 \%$ CIs).

The time to treatment discontinuation was defined as the time from anticancer treatment start to treatment withdrawal. The statistical difference between time to treatment discontinuation in patients discontinued due to VTE and patients discontinued due to other AEs was assessed by a log-rank test and the Kaplan-Meier plot was provided.

A comparison between patients experiencing VTE and patients not experiencing VTE was performed for time to progression and objective response rate. Time to progression in the two cohorts was calculated by applying the Kaplan-Meier analysis and the relevant survival curves were generated and compared with the results of the log-rank test. Finally, the $\chi^{2}$ test was used to compare the proportion of patients responding to anticancer treatment in the two cohorts.

The analysis was performed using SAS (Statistical Analysis System, SAS Institute Inc., Cary, NC, USA, Version 9.2) software.

\section{RESULTS}

The study population consisted of 1415 patients treated with experimental antitumour agents.

Demographic and clinical characteristics of the patients are summarised in Table 1.

The median patients' age was 57.3 years (range: $13-85$ ), $62.8 \%$ of patients were women, $90.7 \%$ were $<70$ years. The most common primary tumours were gynaecologic, sarcomas, gastrointestinal and lung cancers (Table 1). In patients with a diagnosis of metastatic disease, which represented $>80 \%$ of the population $(N=1203)$, over $60 \%$ of patients had previously received less than three chemotherapy regimens for advanced disease.

In the study population, the most common schemes included one or more cytotoxic agents only $(49.9 \%)$ and a single antivascular drug (14.6\%).

Among 1415 patients included in the analysis, 56 experienced a VTE event during the period from the first dose to 30 days after treatment discontinuation (maximum follow-up time), with an overall incidence of $3.96 \%$ and a median follow-up of 2 months (range: $0-26$ ). The median time to VTE occurrence was 1.3 months (range: $0-11.3$ ) with $25 \%$ and $75 \%$ of events occurring within 0.7 and 1.9 months, respectively.

The most common events were deep venous thrombosis accounting for $58.9 \%$ of all observed VTEs and pulmonary embolism (23.2\%). Fourteen patients stopped treatment due to VTE and in two cases VTE (pulmonary embolism) resulted in death.

The VTE events were remarkably high in gynaecologic $(6.2 \%)$ as well as in gastric and pancreatic cancer patients (9.6\%) (Table 2). On the opposite patients with sarcoma had the lowest risk to develop VTE $(2.2 \%)$. With respect to the type of treatment, VTE incidence was particularly high in patients treated with cytotoxic agents in combination with an antiangiogenic drug $(8.9 \%)$ as compared with patients treated with other types of treatment, accounting for $\sim 4 \%$ each $(P=0.0344)$. Among gynaecologic and sarcoma patients treated with trabectedin (ET-743) as single agent the incidence of VTE was $8.5 \%$ and $0 \%$, respectively $(P=0.01)$.

At multivariate analysis, after adjusting for gender and age, gynaecologic (HR: 2.8, 95\% CI: 1.29-6.23, $P=0.009$ ) and gastrointestinal tumours (HR 3.23, 95\% CI: $1.18-8.87, P=0.023$ ), cytotoxic plus antiangiogenic agents (HR: 2.6, 95\% CI: 1.11-6.30, $P=0.028$ ), WBC $>11000 \mu \mathrm{l}^{-1}$ (HR: $2.6,95 \%$ CI: $1.10-6.09, P=0.029$ )

Table I Patient's characteristics

\begin{tabular}{lrr}
\hline & N & $\%$ \\
\hline Age $(N=1415)$ & 1284 & \\
$<70$ years & 131 & 90.7 \\
$\geqslant 70$ years & & \\
Gender $(N=1415)$ & 889 & 62.8 \\
Female & 526 & 37.2 \\
Male & & \\
Tumour type $(N=1415)$ & 435 & 30.7 \\
Gynaecologic & 146 & 10.3 \\
Lung & 73 & 5.2 \\
Pancreas/stomach & 182 & 12.9 \\
Sarcoma & 579 & 40.9 \\
Other tumour type & & \\
& & \\
Treatment type $(N=1415)$ & 706 & 79.9 \\
Only cytotoxic & 318 & 7.9 \\
Only target therapy & 112 & 19.7 \\
Cytotoxic and antiangiogenic & 279 & \\
Cytotoxic and other target agent &
\end{tabular}


Table 2 VTE events according to tumour histotype and treatment type

\begin{tabular}{lrrrrr}
\hline Covariates & $\begin{array}{c}\text { Patients } \\
\text { No }\end{array}$ & $\begin{array}{l}\text { Patients } \\
\text { with VTE }\end{array}$ & $\begin{array}{l}\text { Median time } \\
\text { to VTE (days) }\end{array}$ & P-value \\
\hline Tumour type & & & & & \\
$\quad$ Gynaecologic & 435 & 27 & 6.2 & 45 & 0.0028 \\
$\quad$ Lung & 146 & 5 & 3.4 & 7 & \\
Pancreas/stomach & 73 & 7 & 9.6 & 23 & \\
$\quad$ Sarcoma & 182 & 4 & 2.2 & 51 & \\
$\quad$ Other tumour type & 579 & 13 & 2.2 & 47 & \\
& & & & & \\
Treatment type & & & & & \\
$\quad$ Only cytotoxic & 706 & 22 & 3.1 & 48.5 & 0.0344 \\
$\quad$ Only target therapy & 318 & 12 & 3.8 & 16.5 & \\
$\quad$ Cytotoxic and & 112 & 10 & 8.9 & 37.5 & \\
$\quad$ antiangiogenic & & & & & \\
Cytotoxic and other & 279 & 12 & 4.3 & 46.5 & \\
$\quad$ target agent & & & & & \\
\hline
\end{tabular}

Abbreviation: $\mathrm{VTE}=$ venous thromboembolism.

Table 3 Multivariate Cox Proportional Hazard Model of VTE events with age, sex, tumour histotype, treatment type, platelets, haemoglobin and WBC as covariate variables

\begin{tabular}{|c|c|c|c|c|}
\hline Covariates & $\chi^{2}$ & $P$-value & $\begin{array}{l}\text { Hazard } \\
\text { ratio }\end{array}$ & 95\% HR \\
\hline \multicolumn{5}{|l|}{ Sex } \\
\hline Female & 0.2742 & 0.6005 & 1.220 & $0.580-2.565$ \\
\hline Male & - & - & I & - \\
\hline \multicolumn{5}{|l|}{ Age (years) } \\
\hline$\geqslant 70$ & 0.1358 & 0.7125 & 1.169 & $0.510-2.677$ \\
\hline$<70$ & - & - & 1 & - \\
\hline \multicolumn{5}{|l|}{ Tumour histoype } \\
\hline Gynaecologic & 6.7467 & 0.0094 & 2.836 & $1.292-6.228$ \\
\hline Lung & 1.7066 & 0.1914 & 2.019 & $0.704-5.792$ \\
\hline Pancreas/stomach & 5.1764 & 0.0229 & 3.230 & $1.176-8.867$ \\
\hline Sarcoma & 0.0113 & 0.9154 & 1.064 & $0.340-3.329$ \\
\hline Other tumour type & - & - & I & - \\
\hline \multicolumn{5}{|l|}{ Treatment type } \\
\hline Only target therapy & 2.4353 & 0.1186 & 1.768 & $0.864-3.615$ \\
\hline $\begin{array}{l}\text { Cytotoxic and } \\
\text { antiangiogenic }\end{array}$ & 4.8096 & 0.0283 & 2.644 & 1.109-6.305 \\
\hline $\begin{array}{l}\text { Cytotoxic and other } \\
\text { target agent }\end{array}$ & 0.0230 & 0.8796 & 0.944 & $0.447-1.995$ \\
\hline Only cytotoxic & - & - & । & 1 \\
\hline \multicolumn{5}{|l|}{ Platelets } \\
\hline$\geqslant 350 \times 10 E 9 L^{-1}$ & 0.0782 & 0.7798 & 0.910 & $0.469-1.764$ \\
\hline$<350 \times 10$ E9 $L^{-1}$ & - & - & 1 & I \\
\hline \multicolumn{5}{|l|}{ Haemoglobin } \\
\hline$<100 \mathrm{gL}^{-1}$ & 4.3686 & 0.0366 & 3.098 & $1.073-8.943$ \\
\hline$\geqslant 100 \mathrm{gL}^{-1}$ & - & - & 1 & 1 \\
\hline \multicolumn{5}{|l|}{ WBC } \\
\hline$>11 \times 10 E 9 L^{-1}$ & 4.7733 & 0.0289 & 2.592 & $1.103-6.090$ \\
\hline$\leqslant 11 \times 10 E 9 L^{-1}$ & - & - & I & 1 \\
\hline
\end{tabular}

Abbreviations: $\mathrm{HR}=$ hazard ratio; $\mathrm{VTE}=$ venous thromboembolism; $\mathrm{WBC}=$ white blood cells.

and haemoglobin $<10 \mathrm{~g} \mathrm{dl}^{-1}$ (HR: 3.1, 95\% CI: 1.07-8.94, $P=0.037)$ were statistically correlated with VTE development (Table 3).

The flow chart showing patient disposition until treatment discontinuation is reported in Figure 1. The most common reason for drug discontinuation was the progression of the disease $(63.9 \%$ of patients), followed by $\mathrm{AE}(16.3 \%)$. Among this latter subgroup, in $6.1 \%$ of the cases VTE was the only reason for drug discontinuation.

The AEs causing most frequently drug discontinuation are shown in Figure 2.
Bone marrow toxicity was the most common reason $(45.2 \%)$ followed by peripheral neurological toxicity (7.8\%). Venous thromboembolism was the fourth most common $\mathrm{AE}$ requiring drug discontinuation (Figure 2).

The median time from the first drug administration to treatment discontinuation was statistically different for withdrawals due to VTE as compared with withdrawals due to other AEs: 1.4 and 2.3 months, respectively $(P=0.02)$ (Figure 3 ).

The analysis of response rate comparing patients with and without VTE showed a response rate of $12.5 \%$ and $8.9 \%$, respectively $(P=0.47)$. Likewise, time to progression did not differ between the two cohorts (Figure 4). Response rate and time to progression cohorts were generated by matching each patient with VTE with two patients without VTE according to tumour type, age $( \pm 5)$, gender and treatment type; perfect matching was achieved in $91(81.3 \%)$ of the controls randomly selected, in the other cases, matching between patients with/without VTE was done according to three or two of the variables listed above.

\section{DISCUSSION}

Thrombosis is a leading complication in advanced cancer representing a major health burden for patients and care providers. Our data clearly demonstrate that $4 \%$ of cancer patients treated within the context of early phase anticancer experimental treatments, including target therapies, will develop a VTE episode and, as a consequence, stop treatment, negatively impacting on the results of the studies in which they were enrolled. In this study, we further refine the search for VTE predictors in this special patient's population to provide guidance for the prophylaxis of specific high-risk subgroups. The remarkably high incidence observed in gynaecologic and gastric/pancreatic cancer patients in our series matches well with a previous report (Vemulapalli et al, 2009), observing a VTE incidence of $11.8 \%$. In our series, the role of tumour histotype is well supported by the difference in VTE rate observed in gynaecologic tumours and in sarcoma patients receiving the same antitumour drug, trabectidin. The VTE incidence in gynaecologic tumours patients was $8.5 \%$ and $0 \%$ in the sarcoma patients, respectively $(P=0.01)$.

The combined use of an antiangiogenic plus a cytotoxic agent also identifies a group of subjects with a high risk of developing VTE (8.9\% vs $3.5 \%$ of patients treated with other regimens). Again this increased risk is consistent with the results reported in phase III studies combining a cytotoxic with an antiangiogenic (Kabbinavar et al, 2009) as well as in previous meta-analyses (Nalluri et al, 2008; Cao et al, 2009). There are no hints that the higher incidence observed in this subgroup could be explained by a longer time on study implying a higher probability to observe a VTE, since the median observation period of this group $(2.2$ months) does not show any significant difference as compared with that of the other antitumour regimens (2.0 months) and considering also that $75 \%$ of the observed VTEs occurred within 1.9 months after treatment start.

In our study, we also found an association of leukocytosis with VTE. Several reasons may justify these findings: it is well known that there is a cross-talk between granulocytes and platelets and/or endothelial cells; furthermore, in cancer patients, leukocytosis may also be a surrogate for advanced disease burden not captured by stage classification.

In our series, VTE was the fourth most common AE as cause of drug discontinuation $(6.1 \%)$, being bone marrow toxicity the most frequent reason for treatment withdrawal (45.2\%) followed by peripheral neurological toxicity $(7.8 \%)$, infection and liver function abnormalities (7\% each). Of notice the observation that VTE was more frequent than neuropathic pain, nausea/vomiting, diarrhoea, renal function abnormalities, hand foot syndrome and 


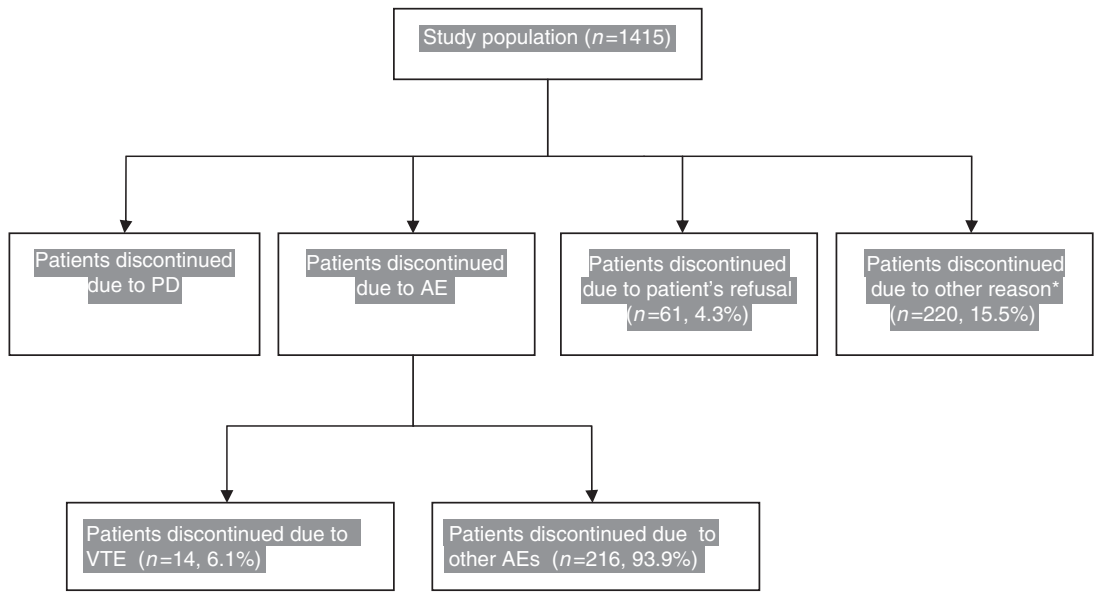

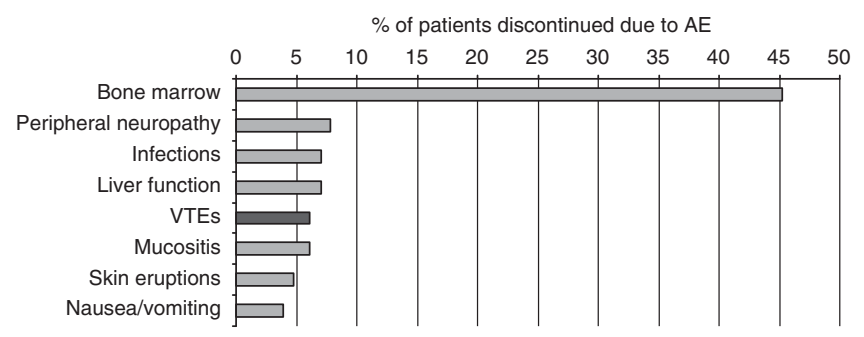

Figure 2 Adverse event as cause of treatment discontinuation.

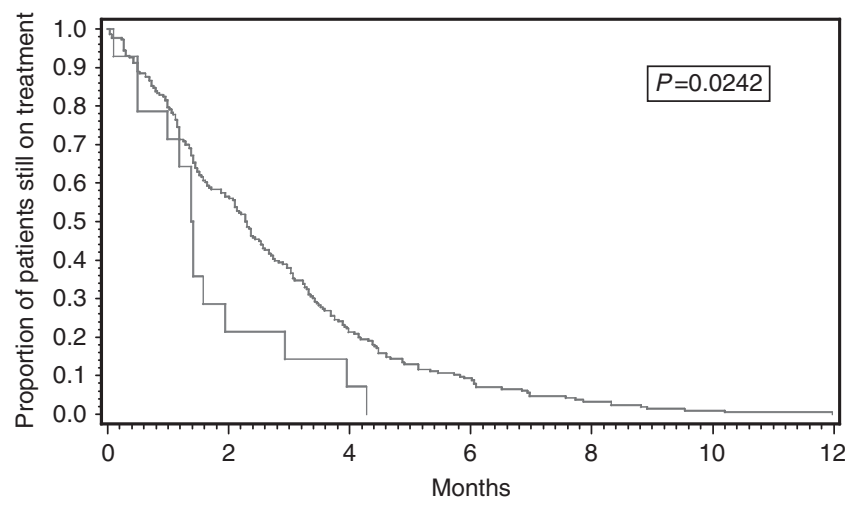

Patients discontinued due to other AEs — Patients discontinued due to VTE

Figure 3 Kaplan-Meier curve of time to treatment discontinuation (only for patients discontinued due to AEs). P-value for the log-rank test is shown.

other skin toxicities, generally perceived by the oncologists as toxicities particularly worthy of attention.

In a recent review evaluating how DLTs have been defined in oncology phase I trials of molecular target agents, VTE was never found to be part of any DLT definition (Le Tourneau et al, 2011). Likewise, VTE is not reported as toxicity in most of the trials evaluating cytotoxic drugs. In a recent European Drug Development Network (EDDN) collaboration study (14), including high quality data from 2232 patients treated in the context of phase I studies in 14 major European institutions between 2005 and 2007, VTE was not reported in most of the considered trials.

All the above data set well the scene regarding the underestimation of these events in the context of new drug development and raise the issue of a more adequate collection of toxicity data in

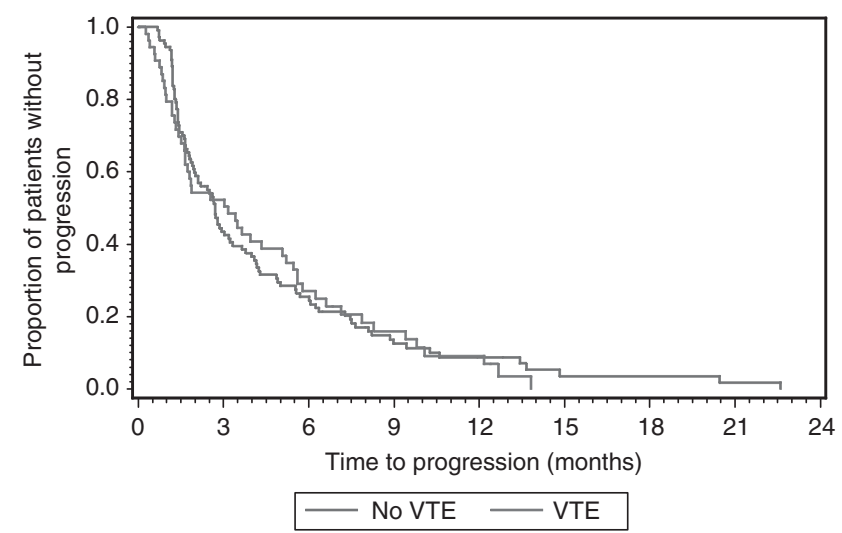

Figure 4 Kaplan-Meier curve of time to disease progression.

order to well describe one of the most important causes of morbidity in cancer patients.

We are not able to comment on awareness per se and neither on attitude to VTE, since these would need qualitative methodologies to investigate. Further studies are needed to clarify this issue within the oncology community.

The strength of this study is its relative large cohort of patients, all treated in SENDO centres, with prospective safety data collection; on the other hand, a limitation is that by selecting only symptomatic, objectively diagnosed VTEs, a number of asymptomatic VTEs may have been missed with a possible consequent underestimation of the risk of this type of event. Furthermore, we did not investigate the role of the D-dimer and available thrombin generation. A prospective randomised study was carried out in 32 metastatic breast cancer patients receiving chemotherapy (Falanga et al, 1998). In this study, the D-dimer was elevated in cancer patients as compared with normal controls and declined in 16 patients receiving warfarin prophylaxis. Nevertheless, none of the laboratory variables could predict thrombosis in individual patients. Furthermore, it should be taken into consideration that in the above-mentioned EDDN trial (Cassier et al, 2011) in 2 years $>2000$ patients entered phase I trials: these figures demonstrate that patients entering phase I studies are not a niche among cancer patients.

Although in this study 137 patients (9.7\%) received at least one thromboprophylactic dose of low molecular weight heparin, in absence of VTE, it was not possible to evaluate the impact of anticoagulant thromboprophylaxis mainly because of the heterogeneity with regards to the beginning and the duration of thromboprophylaxis. 
The median time from the first drug administration to treatment discontinuation was statistically different for patients withdrawn due to VTE as compared with patients withdrawn due to other AEs: 1.4 and 2.3 months, respectively $(P=0.02)$ (Figure 3 ), thus most of these events occur while patients are on treatment and lead to earlier treatment discontinuation. The clinical implication of these findings is that VTE may represent a cause of premature withdrawal of a potentially active drug in cancer patients.

In summary our data demonstrate that, within the context of early clinical trials of new anticancer drugs, VTE is far from being a negligible toxicity and represents the fourth and earliest cause of treatment discontinuation. While no causative explanations can be derived from a purely clinical database such ours, the strength of the relationship between VTE occurrence and specific tumours types, treatments combinations and tumour burden-related conditions suggest that these patients have a much higher chance of developing VTE than other patients. Since their risk is comparable to that observed in other high-risk settings such as that of the hospitalised, medically ill patients for whom prophylaxis is recommended by international guidelines (Lyman et al, 2007; Mandala et al, 2010), should these results be confirmed in other series, primary thromboprophylaxis should be given to them as well. Finally, our results pose the question of VTE underestimation in the current literature and in the planning of early clinical trials.

\section{REFERENCES}

Cao Y, Tan A, Gao F, Liu L, Liao C, Mo Z (2009) A meta-analysis of randomized controlled trials comparing chemotherapy plus bevacizumab with chemotherapy alone in metastatic colorectal cancer. Int J Colorectal Dis 24: 677-685

Cassier PA, Moreno Garcia V, Gomez-Roca C, Olmos D, Morales R, Del Conte G, Gallerani E, Brunetto A, Schoffski P, Marsoni S, Schellens JHM, Penel N, Voest EE, Evans TRJ, Plummer R, Wilson RH, Soria J, Tabernero J, Verweij J, Kaye SB, European Drug Development Network (2011) Doseresponse relationship in phase I clinical trials: a European Dose Drug Development Network (EDDN) collaboration study Proceedings of the American Society of Clinical Oncology. J Clin Oncol 29(Suppl): Abstr 3084

Falanga A, Levine MN, Consonni R, Gritti G, Delaini F, Oldani E, Julian JA, Barbui T (1998) The effect of very-low-dose warfarin on markers of hypercoagulation in metastatic breast cancer: results from a randomized trial. Thromb Haemost 79: 23-27

Falanga A, Zacharski L (2005) Deep vein thrombosis in cancer: the scale of the problem and approaches to management. Ann Oncol 16: 696-701

Kabbinavar FF, Hurwitz HI, Yi J, Sarkar S, Rosen O (2009) Addition of bevacizumab to fluorouracil-based first-line treatment of metastatic colorectal cancer: pooled analysis of cohorts of older patients from two randomized clinical trials. J Clin Oncol 27: 199-205

Kahn SR, Ducruet T, Lamping DL, Arsenault L, Miron MJ, Roussin A, Desmarais S, Joyal F, Kassis J, Solymoss S, Desjardins L, Johri M, Shrier I (2005) Prospective evaluation of health-related quality of life in patients with deep venous thrombosis. Arch Intern Med 165: 1173-1178

Le Tourneau C, Razak AR, Gan HK, Pop S, Diéras V, Tresca P, Paoletti X (2011) Heterogeneity in the definition of dose-limiting toxicity in phase I

cancer clinical trials of molecularly targeted agents: a review of the literature. Eur J Cancer 47: 1468-1475

Lyman GH, Khorana AA, Falanga A, Clarke-Pearson D, Flowers C, Jahanzeb M, Kakkar A, Kuderer NM, Levine MN, Liebman $H$, Mendelson D, Raskob G, Somerfield MR, Thodiyil P, Trent D, Francis CW, American Society of Clinical Oncology (2007) American Society of Clinical Oncology guideline: recommendations for venous thromboembolism prophylaxis and treatment in patients with cancer. J Clin Oncol 25: 5490-5505

Mandala M, Falanga A, Roila F (2010) Management of venous thromboembolism in cancer patients: ESMO clinical recommendations. Ann Oncol 19(Suppl 2): ii126-ii127

Mandala M, Clerici M, Corradino I, Vitalini C, Colombini S, Torri V, De Pascale A, Marsoni S (2012) Incidence, risk factors and clinical implications of venous thromboembolism in cancer patients treated within the context of phase I studies: the 'SENDO experience'. Ann Oncol 23: $1416-1421$

Maur M, Sessa C. Review: new biological insights in early clinical studies (2007) Hematol Oncol 25: 53-57

Nalluri SR, Chu D, Keresztes R, Zhu X, Wu S (2008) Risk of venous thromboembolism with the angiogenesis inhibitor bevacizumab in cancer patients: a meta-analysis. JAMA 300: 2277-2285

Vemulapalli S, Chintala L, Tsimberidou AM, Dhillon N, Lei X, Hong D, Kurzrock R (2009) Clinical outcomes and factors predicting development of venous thromboembolic complications in patients with advanced refractory cancer in a phase I clinic: the M. D. Anderson Cancer Center experience. Am J Hematol 84: 408-413

This work is published under the standard license to publish agreement. After 12 months the work will become freely available and the license terms will switch to a Creative Commons Attribution-NonCommercial-Share Alike 3.0 Unported License. 